\title{
Numerical analysis of TDS spectra under high and low flux plasma exposure conditions
}

\author{
P. Grigorev ${ }^{1,4}$, L. Buzi ${ }^{3-6}$, A. Bakaeva ${ }^{1,4}$, D. Terentyev ${ }^{1}$, G. De Temmerman ${ }^{2}$, G. Van Oost ${ }^{4}$ and J- \\ M. Noterdaeme ${ }^{4}$
}

${ }^{1}$ SCK $•$ CEN, Nuclear Materials Science Institute, Boeretang 200, 2400 Mol, Belgium

${ }^{2}$ ITER Organisation, Route de Vinon-sur-Verdon - CS 90046 - 13067 St Paul Lez Durance Cedex France

${ }^{3}$ FOM Institute DIFFER, Edisonbaan 14, 3439 MN Nieuwegein, The Netherlands

${ }^{4}$ Department of Applied Physics, Ghent University, St. Pietersnieuwstraat 41, 9000 Ghent, Belgium

${ }^{5}$ Institut für Energie und Klimaforschung - Plasmaphysik, Forschungszentrum Jülich GmbH, LeoBrandt-Straße, 52425 Jülich, Germany

${ }^{6}$ Université de Lorraine, Institut Jean Lamour, CNRS UMR 7198, Bvd. des Aiguillettes, F-54506

Vandoeuvre, France

E-mail: pgrigore@sckcen.be

\begin{abstract}
Recently developed numerical model, based on the dislocation-driven nucleation of gas bubbles, is used to analyse experimental results on deuterium retention in tungsten under ITER relevant plasma exposure conditions. Focus is put on understanding the relation between exposure temperature and flux on primary features of thermal desorption spectra: peak positions and intensities of the desorption flux. The model allows one to relate the peak positions with the size of plasma induced deuterium bubbles and envisage exposure conditions (temperature and flux) for their formation. Based on the performed analysis, dedicated experimental conditions to validate the model are proposed.
\end{abstract}

Keywords: hydrogen, retention, plasma, blisters, bubbles.

\section{Introduction}

Development and qualification of plasma facing materials is one of the main challenges in designing large-scale fusion devices(i.e. ITER and DEMO). Due to its favorable physical properties, tungsten (W) is considered to be one of the main candidate materials and is chosen for divertor armour in ITER [1]. The latter should be able to withstand severe conditions in terms of heat and particle loads without considerable degradation of its mechanical and thermal properties within scheduled operational time. Moreover, the ITER nuclear licensing imposes limits on the amount of tritium making the problem of hydrogen isotope (hereinafter referred to as "hydrogen" or " $H$ ") retention an additional technological challenge.

$\mathrm{H}$ retention is expressed in surface modification, formation of subsurface blisters and accumulation of $\mathrm{H}$ in the bulk material. These effects are attributed to trapping of $\mathrm{H}$ atoms on lattice defects such as vacancies, dislocations/grain boundaries and voids [2-4]. Typical kinetic energy of $\mathrm{H}$ ions coming from plasma (below $500 \mathrm{eV}$ ) is far below the threshold energy needed to create a stable Frenkel-pair 
defect in $\mathrm{W}$, and the implantation range of $\mathrm{H}$ ions is limited to several nanometers [5]. Nevertheless, experiments with ion beams and linear plasma generators show that $\mathrm{H}$ penetrates up to a depth of several $\mu \mathrm{m}$ [6-8]. H retention under such conditions cannot be assigned to vacancies since the thermal concentration of vacancies is negligible and no vacancies are created during plasma exposure directly via displacement damage. However, Thermal Desorption Spectroscopy (TDS) demonstrates three typical release stages, attributed to static traps in the material or also called "natural traps":

dislocations, grain boundaries, vacancies and voids [8]. The formation of the vacancy-like trapping defects with subsequent bubble nucleation/growth and blistering under sub-threshold exposure conditions was proposed to originate from the dynamic plasma-induced material modification in the vicinity of a trapping lattice defect, namely: a screw dislocation line.

An alternative model of plasma-induced dislocation-driven bubble formation was proposed recently on the basis of $a b$ initio calculations [9]. It was demonstrated that $\mathrm{H}$ atoms are attracted to a screw dislocation core and exhibit fast one-dimensional migration along the dislocation line. However, the binding energy of this interaction of $0.5 \mathrm{eV}$ is not high enough to keep $\mathrm{H}$ atoms trapped at experimental temperature range (usually above $400 \mathrm{~K}$ ). According to the calculations, once a cluster of eight $\mathrm{H}$ atoms is formed, it should spontaneously transform into a vacancy-like defect (jog) on a dislocation line, and this defect traps $\mathrm{H}$ with a considerably higher binding energy. Thus, dislocations (and dislocation junctions) are suggested to act as nucleation sites for stable $\mathrm{H}$ bubbles. The interaction strength of $\mathrm{H}$ with a newly formed jog on a dislocation is very similar to vacancy- $\mathrm{H}$, as was confirmed by $a b$ initio calculations in [9]. Further growth of $\mathrm{H}$-jog clusters and accumulation of vacancy jogs will lead to the formation of a nanometric cavity-like defect. Theses defects will exhibit extended open volume, and thus, $\mathrm{H}$ interaction with such traps will be similar to nano-voids and cavities. The role of dislocations in bubble formation was validated in recent experiments involving the plasma exposure of annealed and plastically deformed $\mathrm{W}$ samples $[9,10]$. Based on the dislocation-driven nucleation, a numerical simulation tool was developed and used to predict the conditions for the bubble formation depending on exposure temperature and flux [11]. However under the conditions where dislocationdriven mechanism is not active, other microstructural features may act as nucleation sites for bubble nucleation. For example in work [12], the observation of bimodal size distribution of blisters was attributed to an alternative mechanisms - cavity growth and coalescence on grain boundary interfaces.

In Ref [13], the effect of exposure flux - temperature on surface modification and TDS spectra was studied by a set of experiments varying the flux and exposure temperature, respectively, in the range of $9 \times 10^{21}-5 \times 10^{23} \mathrm{D} / \mathrm{m}^{2} / \mathrm{s}$ and $530-870 \mathrm{~K}$. The goal of this work is to estimate $\mathrm{H}$ release from $\mathrm{H}-\mathrm{jog}$ and void-like (multiple vacancy jogs) defects formed during the exposure by successive jog-punching events in support of interpretation of the desorption spectra. We propose a parameterization of the binding energy, defining the $\mathrm{H}$ release based on $a b$ initio results for $\mathrm{H}$ interaction with jogs and cavities as limiting cases. For describing the binding of $\mathrm{H}$ to jogs, and jog clusters (in transition state from jogs to bubbles), we use size-dependent fitting. By comparing the release rate, computed following our model, with experimental TDS spectra we establish a relation between positions of release stages and mean size of the defects releasing $\mathrm{H}$.

\section{Model description}

Following the above mentioned dislocation-driven model of $\mathrm{H}$ trapping, one can distinguish the formation of four types of defects: (I) sub-critical $\mathrm{H}$ clusters (up to eight $\mathrm{H}$ ), which are not yet capable to punch out W matrix atoms; (II) super-critical $\mathrm{H}$ clusters, whose transformation into jog- $\mathrm{H}_{\mathrm{N}}$ configuration is energetically favorable; (III) large super-critical clusters, which already released several jogs for itself and grow further; and finally (IV) nanometric bubble attached to a dislocation 
line, whose properties (in terms of $\mathrm{H}$ trapping) are equivalent to the usual bubble in $\mathrm{W}$ bulk. Here, we will not consider the trapping and release from the defects of type I, since these clusters are unstable above $450 \mathrm{~K}$, while the latter being the lower bound of the relevant temperature range.

In work [3], a rate theory model of $\mathrm{H}$ desorption from tungsten surface and subsurface defects demonstrated that $\mathrm{H}$ release at temperatures, higher than $600 \mathrm{~K}$ is not affected by surface recombination. Here, we deal with assessment of high temperature desorption stage (above 600K), and therefore assume an immediate release of $\mathrm{H}$ from the surface after detrapping from the nano-cavities and vacancies. The main parameter of the model that defines the release rate of $\mathrm{H}$ from a certain type of a trapping defect is the binding energy $\left(E_{b}\right)$. As mentioned before, ab initio calculations showed that dislocation jogs exhibit trapping properties similar to vacancies [9]. The corresponding value of the binding energy is $1.24 \mathrm{eV}$ for one $\mathrm{H}$ atom and it decreases with an increase of $\mathrm{H}$ atoms trapped at the jog. The dependence of $\mathrm{H}_{N}$-jog binding energy on $N$ in a cluster is plotted in Fig. 1. We fitted these data points to generate a family of $E_{b}$ functions for super-critical clusters. The release rate from such defects is defined as:

$$
R_{J}^{-}=C_{H} v \exp \left(-\frac{E_{b}+E_{m}}{k T}\right)
$$

where $C_{H}$ - concentration of trapped $\mathrm{H}$ atoms (assuming that each $\mathrm{H}$ in a cluster has equal probability for detrapping event), $v$ - Debye frequency, $E_{b}$ - binding energy, $E_{m}$ - migration energy $(0.4 \mathrm{eV}$ is taken from Frauenfelder's data [14]), $k$ - Boltzmann constant, $T$ - temperature.

For large vacancy clusters (i.e. nano-voids, type IV defects in the above notation) it is reasonable to consider that the binding energy is equal to the permeation energy, i.e. $\sim 2.0 \mathrm{eV}$ [15]. Under this assumption, one can assign the high temperature TDS peak (usually seen in low flux exposures around $700-900 \mathrm{~K}$ ) to the release from bubbles [16]. However, the TDS spectra obtained after the high flux exposures, at high temperature (above $600 \mathrm{~K}$ ) and/or high dose (above $10^{26} \mathrm{D} / \mathrm{m}^{2}$ ), reveal broadening or presence of extra peaks above $900 \mathrm{~K}[13,17]$. These high temperature release stages cannot be simply explained by detrapping from voids with the binding energy of $2.0 \mathrm{eV}$, while there are no reasons to assume the presence of any stronger traps than voids/cavities.

To address the issue of high temperature detrapping stage, we analyse the information available from atomic scale. Ab initio and Molecular Dynamics simulations showed that $\mathrm{H}$ tends to occupy off-centred position in a vacancy, experiencing weak attraction to $\mathrm{W}$ atoms $[18,19]$. This implies that $\mathrm{H}$ atoms, when filling a void, first should occupy the inner surface positions and then fill the centre.

Thus for the binding energy corresponding to the $\mathrm{H}$ trapped at nm size bubbles (type IV defects) we used the results of ab initio calculations to account for the transition of $\mathrm{H}$ atom from adsorbed state on $\mathrm{W}$ surface into the bulk material [15]. These calculations reported $1.96 \mathrm{eV}$ as a value of the binding energy and attempt frequency of $1.07 \times 10^{13} \mathrm{~s}^{-1}$. However, the expression for the release rate should be different from Eq. 1, since only atoms in adsorbed state on the inner surface of the bubble are available for the detrapping reaction. Thus, the release from nm-scale bubbles is defined by:

$$
R_{B}^{-}=C_{T} N_{S} v_{a d s} \exp \left(-\frac{E_{b}^{a d s}+E_{m}}{k T}\right)(2)
$$

where $C_{T}$ - trap concentration, $N_{S}$ - number of available sites for the reaction, we estimate this as a ratio between an inner surface area of the bubble and unit surface for the reaction reported in [15] ( $\sqrt{2} a_{0}^{2}, a_{0}$ - lattice unit), $v_{a d s}, E_{b}^{a d s}$ attempt frequency and binding energy from [15]. To create a universal binding energy master curve accounting for a size of the trapping defect (i.e. number of vacancies released), we propose the following expression: 


$$
R^{-}=R_{B}^{-}+\left(R_{J}^{-}-R_{B}^{-}\right) N_{V}^{-1 / 3}(3)
$$

here $R_{B}^{-}$and $R_{J}^{-}$are the release rates for bubble and jog correspondingly, $N_{V}$ - number of empty lattice sites in tungsten lattice which the trapping defect occupies. The illustration of the binding energy variation as a function of $N_{V}$ is presented in Fig. 1. To compute the $\mathrm{H}$ release profile from a defect (of a given size and for a given temperature ramp), we assume that after the exposure the defect is filled with $\mathrm{H}$ up to a critical pressure (6.5 $\mathrm{H}$ atoms per $\mathrm{W}$ site), following the analysis done in [11] based on the jog-punching mechanism. Then, the $\mathrm{H}$ release rate from the defect is calculated as a function of increasing temperature. The goal of such calculations is to define the position of the release peak, depending on the size of the defect and concentration of $\mathrm{H}$ inside.

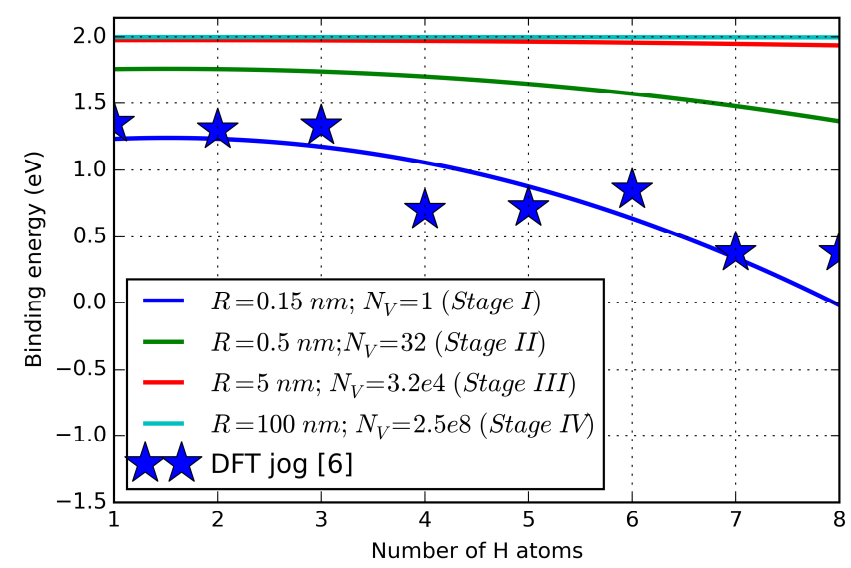

Pic. 1. Illustration of binding energy master curve used in simulations. $R$ - defect open volume radius, $N_{V}$ - corresponding number of W lattice sites that defect occupies.

\section{Results and discussion}

Following the above formulated equations, we analyse the TDS spectra obtained in [13]. In Fig. 2, a comparison of three normalized TDS spectra with the release rate according to the calculations is represented. Normalization of the TDS spectra was done by dividing each one by the maximum release rate value, since here we are interested in correspondence of the peak positions, but not the absolute values of $\mathrm{H}$ release. As can be seen form Fig. 2, TDS spectra for the low flux (fig. b) and high flux high temperature exposure (fig. a) reveals similar single peak as in the spectrum measured after $\sim 870 \mathrm{~K}$ exposure. Temperature position of the peak corresponds to the release from 5-10 nm bubbles, following our calculations. TDS spectrum for high flux low temperature exposure (blue triangles) shows two peaks at lower temperature, whose positions can be well fit by imposing the release rate from $1.5 \mathrm{~nm}$ and $0.5 \mathrm{~nm}$ defects (blue and green lines in Fig. 2a ). 

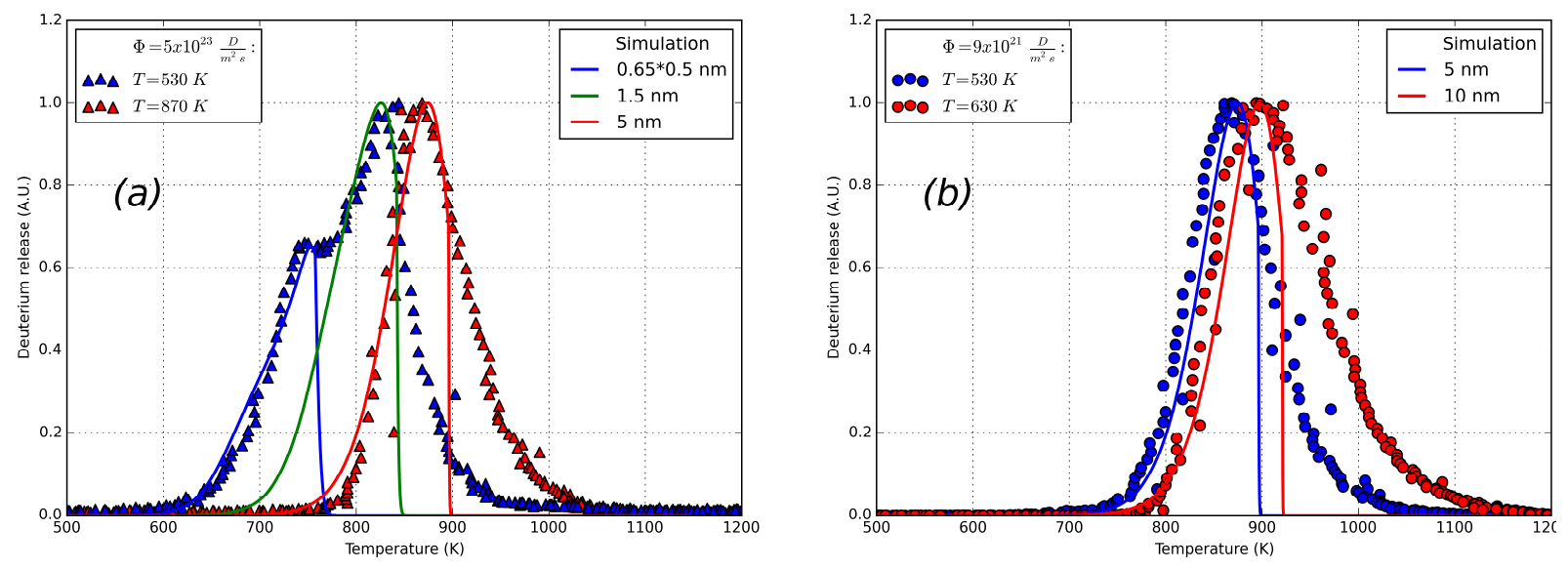

Fig 2. Comparison of normalized TDS spectra from work [13] with results of simulation for high flux $\left(5 \times 10^{23} \mathrm{D} / \mathrm{m}^{2} \mathrm{~s}\right.$ at $530 \mathrm{~K}$ and $\left.870 \mathrm{~K}\right)(\mathrm{a})$ and low flux $\left(9 \times 10^{21} \mathrm{D} / \mathrm{m}^{2} \mathrm{~s}\right.$ at $530 \mathrm{~K}$ and $\left.630 \mathrm{~K}\right)(\mathrm{b})$ exposures.

The difference in shape and peak position of the TDS spectra can be related to a size of releasing defects, and in turn interlinked with the trapping mechanism, governing $\mathrm{H}$ retention depending on exposure conditions. In [11], the prediction of experimental flux - temperature combination for the dislocation-driven $\mathrm{H}$ trapping leading to the formation of super-critical $\mathrm{H}$ clusters (contributing to the TDS release at high temperature) was made. The flux-temperature combination favouring the nucleation of super-critical $\mathrm{H}$ bubbles is represented by a grey area in Fig.3. As one can see, only high flux low temperature exposure is within the range of the dislocation-mediated retention. At the same time, all three other dots are outside the defined area. This is consistent with the fact that only the TDS spectrum after high flux low temperature exposure exhibits two peaks (low temperature peak can be attributed to $1 \mathrm{~nm}$ size defects). Whereas, all other spectra show only one peak corresponding to bubbles of 5-10 nm size. Small ( $1 \mathrm{~nm}$ scale) defects can be seen as traces of bubble nucleation on dislocation and their subsequent growth. While, if only large defects contribute to the TDS spectra, it is very likely that other mechanisms (such as triple junctions, natural porosity, etc) but dislocationdriven trapping govern $\mathrm{H}$ retention and bubble formation.

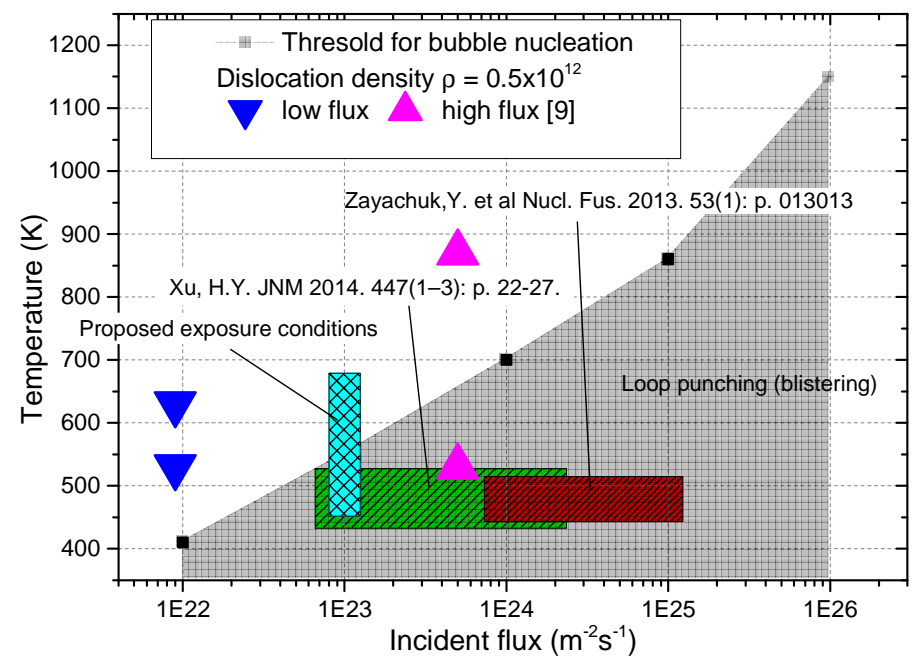

Fig. 3. Comparison of experimental conditions from work [13] with prediction of the model for conditions for dislocation mediated retention.

\section{Conclusive remarks}


The proposed modification for $\mathrm{H}$ release rate from nano-metric pressurized bubbles allows one to relate positions of TDS peaks and explain a shift of high-temperature peak up to $200 \mathrm{~K}$ under exposure conditions favoring the growth of bubbles. The occurrence of a single-peak or multiple-peak in TDS spectra can be related to the mechanism of $\mathrm{H}$ bubble formation, depending on plasma flux and surface temperature. The proposed here computational analysis was applied to experimental data from [13]. According to it, only a combination of high flux and low temperature exposure $\left(530 \mathrm{~K}-5 \times 10^{23} \mathrm{D} / \mathrm{m}^{2}\right)$ promotes the nucleation and growth of $\mathrm{H}$ bubbles on dislocations, as proposed earlier by jog punching mechanism [11]. In these exposure conditions, the TDS spectra exhibit two well defined peaks attributed to $1 \mathrm{~nm}$ size $\mathrm{H}$ clusters (presumably, $\mathrm{H}$ is trapped in jogs on dislocation lines) and $\mathrm{H}$ bubbles (resulting in the high temperature release peak). The three other TDS spectra exhibit only one peak (at high temperature) to be attributed to $\mathrm{H}$ bubbles with a size of 5-10 nm, nucleated at other "natural" traps such as random grain boundaries and their junctions. Validation of the dislocation-driven mechanism and proposed here $\mathrm{H}$ release model can be realized by performing dedicated exposures in $450-600 \mathrm{~K}$ temperature range at flux of $10^{23} \mathrm{D} / \mathrm{m}^{2} / \mathrm{s}$, which should result in the dislocation-driven trapping below $\sim 500 \mathrm{~K}$ to be seen in TDS spectrum as a peak conventionally attributed to vacancy-like defects (i.e. positioned around 600-700 K).

\section{Acknowledgements:}

This work was supported by the European Commission and carried out within the framework of the Erasmus Mundus International Doctoral College in Fusion Science and Engineering (FUSION-DC).

This work has been carried out within the framework of the EUROfusion Consortium and has received funding from the Euratom research and training programme 2014-2018 under grant agreement No 633053. The views and opinions expressed herein do not necessarily reflect those of the European Commission.

\section{References:}

1. Federici, G., et al.,2001, Plasma-material interactions in current tokamaks and their implications for next step fusion reactors. Nuclear Fusion. 41(12): p. 1967.

2. Van Veen, A., et al.,1988, Hydrogen exchange with voids in tungsten observed with TDS and PA. Journal of Nuclear Materials. 155-157, Part 2: p. 1113-1117.

3. Eleveld, H. and A. van Veen,1992, Deuterium interaction with impurities in tungsten studied with TDS. Journal of Nuclear Materials. 191-194, Part A: p. 433-438.

4. Eleveld, H. and A. van Veen,1994, Void growth and thermal desorption of deuterium from voids in tungsten. Journal of Nuclear Materials. 212-215, Part B: p. 1421-1425.

5. Pintsuk, G.,2012, 4.17 - Tungsten as a Plasma-Facing Material. Comprehensive Nuclear Materials: p. 551-581.

6. Hoen, M.H.J.t., et al.,2012, Saturation of deuterium retention in self-damaged tungsten exposed to high-flux plasmas. Nuclear Fusion. 52(2): p. 023008.

7. Schmid, K., V. Rieger, and A. Manhard,2012, Comparison of hydrogen retention in W and W/Ta alloys. Journal of Nuclear Materials. 426(1-3): p. 247-253.

8. Ogorodnikova, O.V., J. Roth, and M. Mayer,2003, Deuterium retention in tungsten in dependence of the surface conditions. Journal of Nuclear Materials. 313-316(0): p. 469-477.

9. Terentyev, D., et al.,2014, Dislocations mediate hydrogen retention in tungsten. Nuclear Fusion. 54(4): p. 042004.

10. Terentyev, D., et al.,2015, Effect of plastic deformation on deuterium retention and release in tungsten. Journal of Applied Physics. 117(8): p. 083302. 
11. Grigorev, P., et al.,2015, Nucleation and growth of hydrogen bubbles on dislocations in tungsten under high flux low energy plasma exposure. Nuclear Instruments and Methods in Physics Research Section B: Beam Interactions with Materials and Atoms. 352(0): p. 96-99.

12. Alimov, V.K., et al.,2012, Temperature dependence of surface morphology and deuterium retention in polycrystalline ITER-grade tungsten exposed to low-energy, high-flux D plasma. Journal of Nuclear Materials. 420(1-3): p. 519-524.

13. Buzi, L., et al.,2014, Influence of particle flux density and temperature on surface modifications of tungsten and deuterium retention. Journal of Nuclear Materials. 455(1-3): p. 316-319.

14. Frauenfelder, R.,1969, Solution and Diffusion of Hydrogen in Tungsten. Journal of Vacuum Science \& Technology. 6(3): p. 388-397.

15. Johnson, D.F. and E.A. Carter,2010, Hydrogen in tungsten: Absorption, diffusion, vacancy trapping, and decohesion. Journal of Materials Research. 25(02): p. 315-327.

16. Ogorodnikova, O.V., et al.,2011, The influence of radiation damage on the plasma-induced deuterium retention in self-implanted tungsten. Journal of Nuclear Materials. 415(1, Supplement): p. S661-S666.

17. Jia, Y.Z., et al.,2015, Surface morphology and deuterium retention in tungsten exposed to high flux D plasma at high temperatures. Journal of Nuclear Materials. 457(0): p. 213-219.

18. Heinola, K., et al.,2010, Hydrogen interaction with point defects in tungsten. Physical Review B: Condensed Matter. 82(9): p. 094102.

19. Sun, L., et al.,2013, Hydrogen behaviors in molybdenum and tungsten and a generic vacancy trapping mechanism for $\mathrm{H}$ bubble formation. Journal of Nuclear Materials. 434(1-3): p. 395401. 